\title{
Ad-VT enhances the sensitivity of chemotherapy-resistant lung adenocarcinoma cells to gemcitabine and paclitaxel in vitro and in vivo
}

\author{
Gaojie Song ${ }^{1,2,3}$ (1) Chao Shang ${ }^{2} \cdot$ Lili Sun $^{4} \cdot$ Yiquan $^{3}{ }^{3} \cdot$ Yilong Zhu $^{3} \cdot$ Zhiru Xiu $^{3} \cdot$ Zirui Liu $^{2} \cdot$ Yaru Li $^{3} \cdot$ Xia Yang $^{3}$. \\ Chenchen $\mathrm{Ge}^{3}$. Jinbo Fang ${ }^{3}$ Ningyi Jin ${ }^{1,2,3} \cdot{\text { Xiao } \mathrm{Li}^{2,3}}^{2,3}$
}

Received: 24 August 2021 / Accepted: 30 November 2021 / Published online: 4 January 2022

(c) The Author(s) 2021

\begin{abstract}
Summary
Background One of the main challenges in the clinical treatment of lung cancer is resistance to chemotherapeutic drugs. P-glycoprotein (P-gp)-mediated drug resistance is the main obstacle to successfully implementing microtubule-targeted tumor chemotherapy. Purpose In this study, we explored the effect of Ad-hTERTp-E1a-Apoptin (Ad-VT) on drug-resistant cell lines and the molecular mechanism by which Ad-VT combined with chemotherapy affects drug-resistant cells and parental cells. Methods In vitro, cell proliferation, colony formation, resistance index (RI), apoptosis and autophagy assays were performed. Protein expression was analyzed by Western blotting. Finally, a xenograft tumor model in nude mice was used to detect tumor growth and evaluate histological characteristics. Results Our results showed that Ad-VT had an obvious killing effect on A549, A549/GEM and A549/Paclitaxel cancer cells, and the sensitivity of drug-resistant cell lines to Ad-VT was significantly higher than that of parental A549 cells. Compared with A549 cells, A549/GEM and A549/Paclitaxel cells had higher autophagy levels and higher viral replication ability. Ad-VT decreased the levels of p-PI3k, p-Akt and p-mTOR and the expression of P-gp. In vivo, Ad-VT combined with chemotherapy can effectively inhibit the growth of chemotherapyresistant tumors and prolong the survival of mice. Conclusions Thus, the combination of Ad-VT and chemotherapeutic drugs will be a promising strategy to overcome chemoresistance.
\end{abstract}

Keywords Lung cancer cells $\cdot$ Oncolytic virus $\cdot$ Apoptosis $\cdot$ Autophagy $\cdot$ P-gp

\section{Introduction}

Gaojie Song

Jinbo Fang

fangjb@ccucm.edu.cn

Ningyi Jin

ningyik@126.com

$\triangle \mathrm{Xiao} \mathrm{Li}$

lixiao06@mails.jlu.edu.cn

Medical College, Yanbian University, Yanji, China

2 Changchun Veterinary Research Institute, Chinese Academy of Agricultural Sciences, Changchun, China

3 Academician Workstation of Jilin Province, Changchun University of Chinese Medicine, Changchun, China

4 Department of Head and Neck Surgery, Tumor Hospital of Jilin Province, Changchun, China
Lung cancer is one of the most common malignant tumors the worldwide. Non-small cell lung cancer (NSCLC) accounts for approximately $80 \%$ of all lung cancers and causes 1.7 million deaths worldwide annually. According to the World Health Organization (WHO), the number of new cases in 2020 was 2,206,771 (11.4\%), the number of deaths was $1796144(18 \%)$. Lung cancer is the leading cause of cancer-related death primarily due to drug resistance, which has always been a major obstacle to successful cancer treatment. The vast majority of patients will gradually develop drug resistance to chemotherapy or targeted drugs even if they show a good initial response [1]. Multidrug efflux is one of the most important mechanisms of chemotherapy resistance and functions by exporting chemotherapeutic drugs from cells, thus protecting them from their antitumor activities [2, 3]. P-glycoprotein (P-gp) is a typical multidrug resistance (MDR) protein that can reduce the intracellular 
concentration of chemotherapy drugs. Therefore, many scientists are investigating ways to alter P-gp function and expression and reverse chemotherapy resistance.

Oncolytic viruses (OVs), which selectively kill tumor cells without exerting any toxic effects on normal cells or tissues, are emerging as important agents in cancer treatment and have shown encouraging safety profiles in clinical trials [4-7]. In previous studies, our team designed and constructed the oncolytic adenovirus Ad-hTERTp-E1a-Apoptin (Ad-VT). Ad-VT contains an E1a gene controlled by the human telomerase reverse transcriptase promoter, which is highly active in more than $85 \%$ of human cancers but not in most normal human cells. In addition, Ad-VT expresses apoptin, a $13.6 \mathrm{kDa}$ protein that is the product of the VP3 gene of chicken anemia virus (CAV) and has the ability to selectively kill a variety of human tumors or transformed cells but little cytotoxic effect on normal cells [8]. Therefore, Ad-VT is a new type of dual-specific oncolytic adenovirus with tumor-specific targeting and killing functions. As an adjuvant and alternative drug, Ad-VT has been studied for its antitumor effect; the results indicate that Ad-VT can inhibit the proliferation of malignant tumor cells in vitro and in vivo. Ad-VT mitigates the growth of tumor cells through a variety of mechanisms, including inducing apoptosis and autophagy, inhibiting the proliferation and differentiation of tumor cells, inducing cell cycle arrest, preventing angiogenesis, and hindering cell energy metabolism. However, the mechanism by which Ad-VT affects drug-resistant lung cancer has not been reported.

This study aims to (1) detect P-gp expression in NSCLC tissues and cell lines and study its biological effects through a series of in vitro and in vivo experiments, (2) evaluate the cytotoxicity of Ad-VT on lung cancer cells in vitro, (3) investigate the mechanism of Ad-VT in chemotherapyresistant lung cancer cells, and (4) determine the therapeutic effect of Ad-VT combined with gemcitabine or paclitaxel in an in vivo lung tumor model.

\section{Materials and methods}

\section{Cells, viruses and animals}

A549 cell lines were purchased from the Cell Bank of the Shanghai Institute for Biological Sciences. A549/GEM and A549/Paclitaxel cell lines were purchased from the $\mathrm{BeNa}$ Culture Collection. The recombinant adenovirus Ad-VT (Ad-hTERTp-E1a-Apoptin) was constructed and preserved in our laboratory (Changchun Veterinary Research Institute, Chinese Academy of Agricultural Sciences, Changchun, China) [9]. Female BALB/c nude mice aged 4 to 5 weeks were purchased from the Experimental Animal Center of the Academy of Chinese Academy of Agricultural Sciences.

\section{Drugs, reagents and antibodies}

Gemcitabine and paclitaxel were purchased from MedChemExpress LLC. Protease inhibitor cocktail and phosphatase inhibitor cocktail were purchased from APExBIO Technology LLC. Primary antibodies against p-mTOR, mTOR, p-AKT, AKT, p-PI3K, PI3K, MDR1, cleaved caspase-3, Beclin-1, LC3A/B and GAPDH were purchased from Cell Signaling Technology, Inc.

\section{Crystal violet staining}

Cells were seeded in 12-well plates, treated with Ad-VT (MOI of 80 ) for $48 \mathrm{~h}$, and then stained with $0.4 \%$ crystal violet for $10 \mathrm{~min}$ at room temperature. The dye solution was discarded, and the stained cells were washed with PBS, dried and imaged.

\section{Hoechst assay}

Cells in the logarithmic growth phase were cultured in 12-well cell culture plates, and $300 \mu$ of Hoechst staining fluid was added to each well and incubated for $15 \mathrm{~min}$. The dye solution was removed, and the cells were air dried and imaged.

\section{Flow cytometric analysis}

Induction of apoptosis was detected by flow cytometry using a FITC Annexin V Apoptosis Detection Kit I. Briefly, cells were seeded in 6-well plates and treated with Ad-VT (MOI of 80 ) for 24,48 , or $72 \mathrm{~h}$. Before analysis, the cells were stained with $5 \mu \mathrm{l}$ of propidium iodide (PI) solution and $5 \mu \mathrm{l}$ of Annexin V-FITC. The samples were then examined by flow cytometry for apoptosis analysis. Each experiment was repeated at least three times.

\section{CCK-8 assay}

The CCK-8 assay was performed as described [10]. Briefly, cells were seeded in 96-well plates and treated with Ad-VT or drugs (gemcitabine and paclitaxel). Then, $10 \mu$ of CCK- 8 solution was added to each well and the cells were incubated for $3 \mathrm{~h}$. Viable cells were assessed using a microplate reader at an absorbance of $595 \mathrm{~nm}$.

\section{Monodansylcadaverine (MDC) detection}

Cells were seeded on microscope cover slips in 6-well plates and cultured for $24 \mathrm{~h}$, after which the medium was discarded. Then, the cells were washed three times with $0.1 \mathrm{~mol} / \mathrm{l}$ of cold PBS before autophagic vacuoles were labeled with 
$0.05 \mathrm{mmol} / \mathrm{l} \mathrm{MDC}$. The percentage of cells with MDClabeled autophagic vacuoles among the total cell population was calculated at $400 \times$ magnification. Cells with 20 or more MDC markers were considered to have positive fluorescence.

\section{Western blot analysis}

Total protein extraction from cells and Western blot analysis were performed as previously described [11, 12]. Protein from cells was extracted using the Minute ${ }^{\mathrm{TM}}$ Total Protein Extraction Kit for Animal Cultured Cells and Tissues. Briefly, $30 \mu \mathrm{g}$ of total protein was separated via SDS/PAGE though $12 \%$ gradient gels and electrotransferred to nitrocellulose membranes. The membranes were blocked with NcmBlot blocking buffer before they were incubated with the appropriate primary antibody at $4{ }^{\circ} \mathrm{C}$ overnight. Then, the membranes were incubated with HRP-conjugated secondary antibody for $40 \mathrm{~min}$ at room temperature and washed with TBST three times. We calculated the band intensities of the proteins using ImageJ software (National Institutes of Health, USA) and normalized them to the intensity of the GAPDH band.

\section{Measurement of viral titers}

A549, A549/GEM and A549/Paclitaxel cells were seeded into 6-well plates at a density of $2 \times 10^{5}$ cells per well $(n=3$ per condition). After $18 \mathrm{~h}$ of incubation, the cells were infected with Ad-VT at 10 MOI. After 24, 48 or $72 \mathrm{~h}$ of infection, the cells and supernatant were collected and then frozen and thawed 3 times. HEK-293 cells were seeded into 96-well plates at a density of $5 \times 10^{3}$ cells per well. After $18 \mathrm{~h}$ of incubation, the cells were infected with tenfold diluted Ad-VT. The virus titers were calculated with the Reed-Muench method and expressed as TCID $_{50}$ per milliliter of supernatant. In the 3-MA-treatment groups, the cells were pretreated with 3-MA for $2 \mathrm{~h}$ before they were subjected to Ad-VT infection.

\section{Analysis of apoptosis by TUNEL staining}

Cells were seeded on microscope cover slips in 6-well plates. After $24 \mathrm{~h}$ of culture, the medium was discarded, and the cells were fixed with immunostaining fixative for 30 min and washed three times with cold PBS. Then, a TUNEL assay was performed using colorimetric TUNEL apoptosis assay kits according to the manufacturer's instructions. Green fluorescence indicates TUNEL-positive cells, and blue fluorescence indicates nuclei with DAPI staining. In addition, a TUNEL assay with tumor sections was performed as previously described [13]. Images were obtained under a fluorescence microscope, and at least three random fields from each section were examined at $200 \times$ magnification.

\section{Xenograft model}

A549, A549/GEM and A549/Paclitaxel cells $\left(1 \times 10^{7}\right)$ were subcutaneously inoculated into the right flank of female $\mathrm{BALB} / \mathrm{c}$ nude mice (4-5 weeks, $20 \pm 2 \mathrm{~g}$ ); 7 days later, these mice were randomly divided into different groups $(n=10$ per group) and then subjected to the indicated treatments. The tumor volume was measured every 5 days with calipers and calculated with the following formula: $[(\mathrm{W} 2 \times \mathrm{L}) / 2 ; \mathrm{W}$, width; L, length; in cubic millimeters]. In the Ad-VT-treated groups, $1 \times 10^{9}$ plaque-forming units of Ad-VT were intratumorally injected in a volume of $100 \mu \mathrm{l}$ in PBS every 3 days for a total of 10 treatments. In the drug-treated groups, gemcitabine $(10 \mathrm{mg} / \mathrm{kg})$ and paclitaxel $(5 \mathrm{mg} / \mathrm{kg})$ in corn oil were intraperitoneally (i.p.) injected in a volume of $100 \mu$ l. The drugs were given every 3 days for a total of 10 treatments. Tumor tissues $(n=3)$ were harvested 30 days after the initial virus injection and fixed in $4 \%$ paraformaldehyde in neutral buffer for $24 \mathrm{~h}$.

\section{Histopathological analysis}

Histopathological analysis of the tissue sections was performed as previously described [14]. Briefly, fixed tumor tissues were embedded in paraffin and sliced to $5 \mu \mathrm{m}$ thickness, after which the sections were blocked with normal horse serum for $1 \mathrm{~h}$ and then incubated with antibodies against $\mathrm{Ki}-67$ or P-gp at $4{ }^{\circ} \mathrm{C}$ overnight. After washing, the sections were incubated with secondary antibodies for $1 \mathrm{~h}$ and then washed with cold PBS. The proteins were visualized by incubating sections with $\mathrm{DAB}$ for $15-20 \mathrm{~min}$ at room temperature followed by counterstaining with hematoxylin. Slices were observed under a light microscope to detect Ki-67 and P-gp expression.

\section{Statistical methods}

All data are presented as the mean \pm SEM unless otherwise indicated, as Pan et al. previously described [15]. Differences between two groups were evaluated for statistical significance using a two-tailed unpaired t-test. When the differences among three or more groups were evaluated, one-way ANOVA was used. Survival data were subjected to Kaplan-Meier survival analysis. A $p<0.05$ indicated statistical significance. 


\section{Result}

\section{Gemcitabine and paclitaxel sensitivity}

A549, A549/Paclitaxel and A549/GEM cell lines were treated with different concentrations of paclitaxel or gemcitabine. The results showed that the $\mathrm{IC}_{50}$ values of paclitaxel in A549, A549/GEM and A549/Paclitaxel cells were $31.441 \pm 1.72,20.22 \pm 1.48$ and $3193 \pm 207.6 \mathrm{~nm} /$ $\mathrm{ml}$, respectively, and the $\mathrm{IC}_{50}$ values of gemcitabine were $481.3 \pm 88.35,8369 \pm 474.6$ and $406.2 \pm 87.11 \mathrm{~nm} / \mathrm{ml}$, respectively (Table 1). The resistance index (RI) of A549/ GEM cells to gemcitabine and paclitaxel was 17 and 1, respectively, and that of A549/Paclitaxel cells to paclitaxel and gemcitabine was 103 and 1, respectively. This finding that A549/GEM cells are sensitive to paclitaxel and that A549/Paclitaxel cells are sensitive to gemcitabine.

\section{P-gp expression was higher in cancer and was associated with shorter overall survival}

$\mathrm{P}$-gp, which reduces the intracellular concentrations of chemotherapeutic agents, is the classical MDR protein. Western blot results demonstrated that P-gp expression in A549/Paclitaxel and A549/GEM cells was significantly higher than that in A549 cells (Fig. 1a). Immunofluorescence staining was used to detect P-gp expression, as shown in Fig. $1 b$, and the results were consistent with the Western blot results. To determine the clinical relevance of P-gp, we analyzed its protein expression in clinical specimens, which was significantly higher in lung adenocarcinoma (LUAD) tissues than in normal lung tissues (Fig. 1c). Furthermore, to assess the clinical significance of $\mathrm{P}$-gp expression in lung cancer, NSCLC patients were further divided into high P-gp expression and low P-gp expression groups based on the mean expression. As shown in Fig. 1d, Kaplan-Meier analysis revealed that high P-gp expression was significantly correlated with poor overall survival in patients with lung cancer.

\section{Ad-VT had a significant inhibitory effect on chemoresistant cell lines}

Ad-hTERTp-E1a-Apoptin (Ad-VT) contains an hTERTpdriven E1a cassette and a CMV-driven Apoptin (VP3 protein) cassette (Fig. 2a). To study the antitumor activity of Ad-VT, A549, A549/GEM and A549/Paclitaxel cells were infected with Ad-VT at different MOIs (twofold dilution from $160 \mathrm{MOI}$ to $0.125 \mathrm{MOI}$ ) for $48 \mathrm{~h}$. Pearson correlation analysis of CCK-8 results showed that the relative survival of various cells was related to the dose of Ad-VT (Fig. 2b).
After infection with Ad-VT (MOI of 80) for 24, 48 and $72 \mathrm{~h}$, the relative survival rates of the three cell lines were significantly decreased in a time-dependent manner (Fig. 2c). The three cell lines were then infected with different concentrations of $\operatorname{Ad-VT}(0,10,20,30,40,80$ and $160 \mathrm{MOI})$ for $48 \mathrm{~h}$. The results showed that compared with A549 cells, Ad-VT cells had obvious cytotoxicity in A549/GEM and A549/ Paclitaxel cells (Fig. 2d). In conclusion, the CCK-8 and crystal violet staining results showed that Ad-VT (MOI of 80) had an obvious killing effect on A549, A549/GEM and A549/Paclitaxel cells after $48 \mathrm{~h}$ of infection, and the killing effect on chemoresistant cells was significantly higher than that on A549 cells. Based on this finding, we used Ad-VT at an MOI of 80 in subsequent experiments. In addition, we observed the replication ability of Ad-VT in cells at different time points. The results showed that the titer of Ad-VT increased in a time-dependent manner in A549, A549/GEM and A549/Paclitaxel cells but that the replication level of Ad-VT in chemoresistant cells was higher than that in A549 cells at the same time points (Fig. 2e).

\section{Ad-VT significantly induced apoptosis in drug-resistant cell lines}

A549, A549/GEM and A549/Paclitaxel cell lines were treated with Ad-VT (MOI of 80), and apoptotic chromatin condensation was examined with Hoechst 33258 staining (Fig. 3a). Ad-VT-induced apoptotic chromatin condensation was obvious in A549/GEM and A549/Paclitaxel cells but minimal in A549 cells. We also assessed the apoptotic effects of Ad-VT through Annexin V-FITC and TUNEL staining (Fig. 3b, c), the results of which were consistent with those of Hoechst 33258 staining. These data indicate that apoptosis was the major mechanism of cell death induced by Ad-VT treatment, which was time dependent.

Quadrant statistical analysis showed that the number of apoptotic chemoresistant cells was significantly higher than the number of apoptotic A549 cells (Fig. 3d). In addition, we noted that Ad-VT significantly increased the expression of PARP and cleaved caspase-3 (Fig. 3e, f). These results indicated that Ad-VT induced caspase family-dependent apoptosis in A549/Paclitaxel and A549/GEM cells. Taken together with previous experiments, our data indicate that Ad-VT induced more significant apoptosis in A549/GEM and A549/Paclitaxel cells than in A549 cells.

\section{Elevated levels of autophagy in chemoresistant cell lines enhanced the effect of Ad-VT}

Autophagy is reported to play an important role in the drug resistance of cancer cells, including NSCLC cells [16]. To detect whether drug-resistant lung cancer cells have high levels of autophagy, Western blot and fluorescence 
a

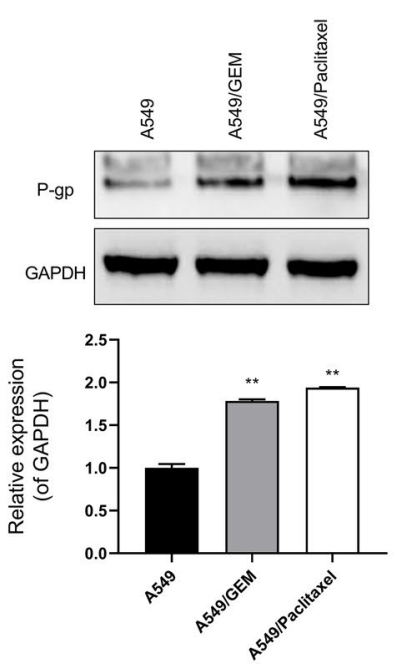

C

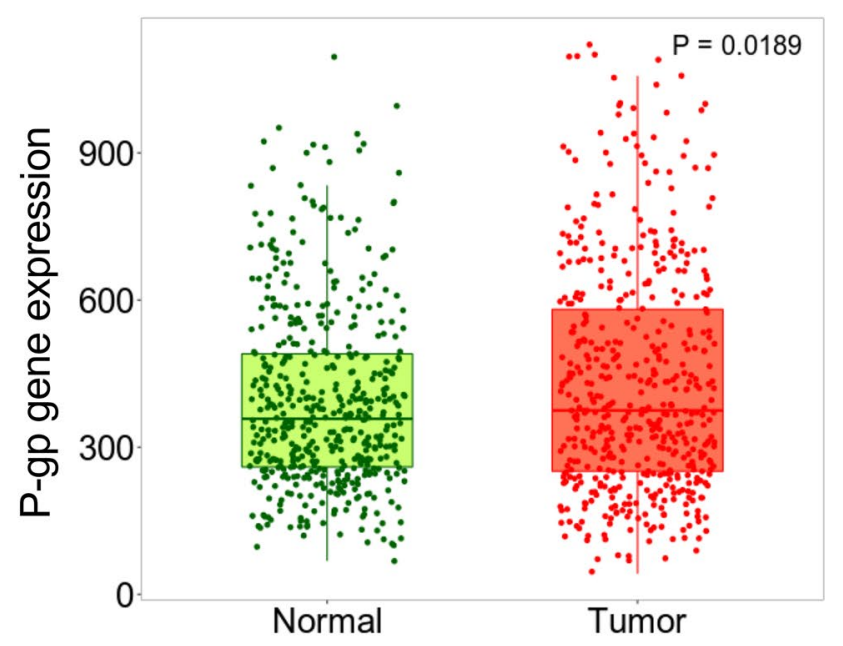

b

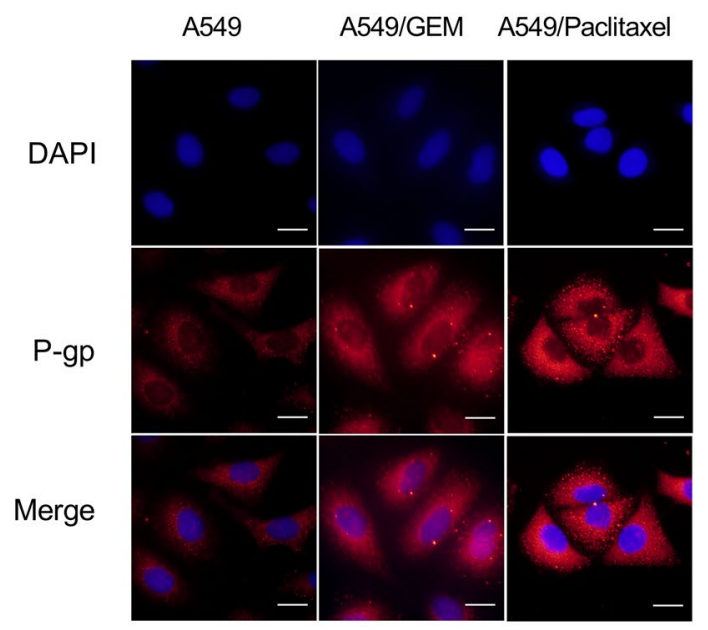

d

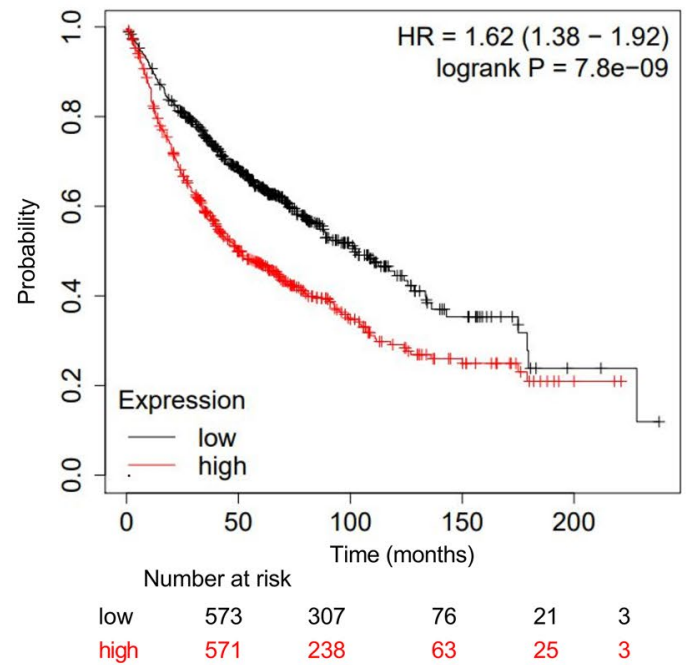

Fig. 1 P-gp is overexpressed in A549/GEM and A549/Paclitaxel cells. a Western blot analysis of P-gp levels in A549, A549/GEM and A549/Paclitaxel cells. b Immunofluorescence analysis of P-gp expression (red fluorescence) and nuclei (blue fluorescence, DAPI staining) in A549, A549/GEM and A549/Paclitaxel cells. Scale bars: $25 \mu \mathrm{m}$. c P-gp expression in normal lung tissue and lung cancer specimens.

microscopy were performed. The results showed that the expression levels of the autophagy-related proteins ATG5, Beclin1, p-mTOR and LC3B in A549/GEM cells were significantly higher than those in A549 cells (Fig. 4a), and the expression trend in A549/Paclitaxel cells was similar to that in A549/GEM cells. The expression of p62 protein in A549/GEM and A549/Paclitaxel cells was significantly lower than that in A549 cells (Fig. 4a). MDC, a known tracer for autophagic vesicles and that emits green fluorescence, is taken up by cells and selectively binds to autophagic vacuoles [17]. The MDC staining results in Fig. 4b show
Images were derived from The Human Protein Atlas (Kaplan-Meier Plotter) online database. d Kaplan-Meier survival curves and log rank tests were established to assess the correlation between P-gp expression and overall survival of lung cancer patients. Each experiment was repeated three times. ${ }^{*} p<0.01$

that punctate MDC-positive fluorescent particles were present in the cytoplasm of A549/GEM or A549/Paclitaxel cells, indicating that the drug-resistant cell lines were more positive for autophagosomes than were A549 cells. When 3-MA, an autophagy inhibitor, was added to A549/GEM and A549/Paclitaxel cells, the number of autophagosomepositive cells was significantly reduced compared with that of A549 cells. These results indicate that chemoresistant lung cancer cells have higher autophagy levels than their parental cells. Finally, we investigated whether autophagy could affect virus replication and, consequently, the effect 
a

Ad-hTERTp-E1a-Apoptin (Ad-VT)

Ad $\mathrm{CMV}$ Apoptin $\mathrm{PA}$ hTERT E1a $\mathrm{PA}$ Ad

b

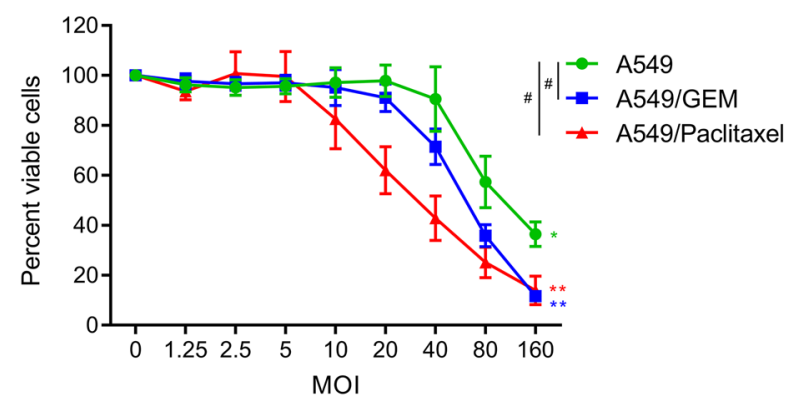

d

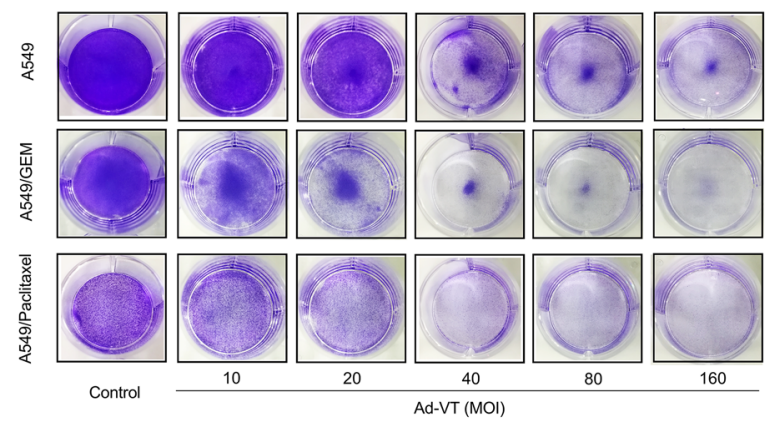

Fig. 2 Comparative efficacy of Ad-VT on the inhibition of lung cancer cell growth and cell viability. a Schematic diagram showing the hTERTp-driven E1a cassette and the CMV-driven-apoptin expressing cassettes of Ad-hTERTp-E1a-Apoptin (Ad-VT). b-c Viability of A549, A549/GEM and A549/Paclitaxel cells as measured using the CCK-8 assay. d Crystal violet staining of cells treated with Ad-VT

of OVs in chemoresistant cells. After $48 \mathrm{~h}$ of treatment with 3-MA, the Ad-VT viral titer in chemoresistant cell lines decreased significantly, suggesting that autophagy significantly increased the level of viral replication in A549/GEM and A549/Paclitaxel cells (Fig. 4d, e and f).

\section{Ad-VT combined with gemcitabine or paclitaxel inhibited the growth and reduced the drug resistance of A549/GEM or A549/Paclitaxel cells}

The effects of Ad-VT and gemcitabine on A549 or A549/ GEM cells were compared using CCK- 8 assay. The results showed that gemcitabine alone could significantly reduce the survival rate of A549 cells but had no significant effect on A549/GEM cells, which supported the resistance of A549/GEM cells to gemcitabine. When gemcitabine was combined with Ad-VT, the survival of A549/GEM cells was significantly lower than that in the gemcitabine alone and
C

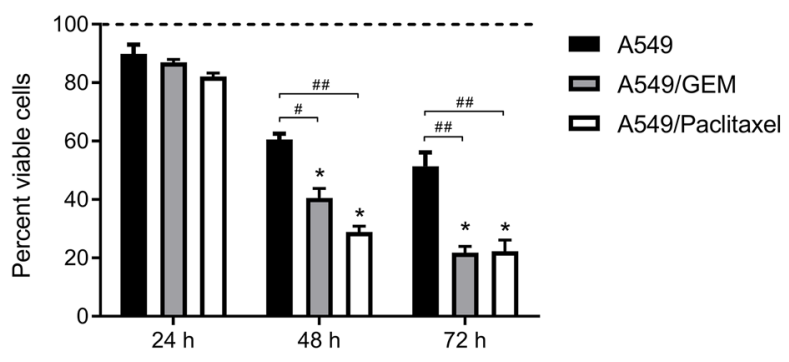

e

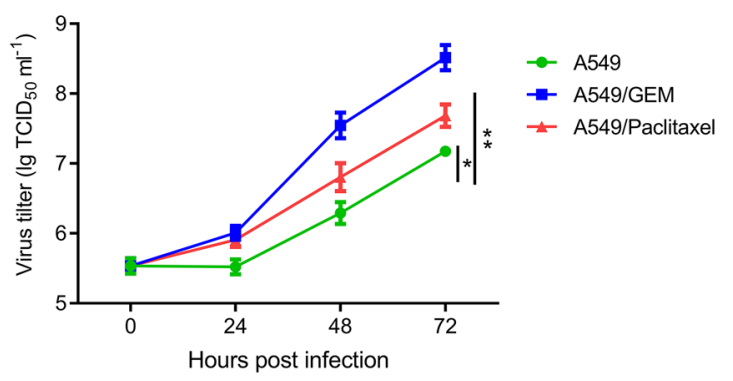

alone at several MOIs (twofold serial dilutions from 160 to 0.125 MOI). e Viral replication in A549, A549/GEM and A549/Paclitaxel lung cancer cells infected with Ad-VT at an MOI of 80 for $24 \mathrm{~h}, 48 \mathrm{~h}$ and $72 \mathrm{~h}$. The data are presented as the means $\pm \mathrm{SD}, \mathrm{n}=3 .{ }^{*} p<0.05$, ${ }^{* *} p<0.01$ compared with the control group. ${ }^{\#} p<0.05,{ }^{\# \#} p<0.01$ compared with the corresponding A549 group

control groups (Fig. 5a). In addition, we studied the effect of Ad-VT combined with paclitaxel on A549/Paclitaxel cells. Paclitaxel significantly decreased the survival of A549 cells but had no significant effect on A549/Paclitaxel cells. However, the combination of Ad-VT and paclitaxel significantly reduced the survival of A549/Paclitaxel cells compared with paclitaxel alone and control treatment (Fig. 5b).

Activation of Akt, a downstream constituent of the PI3K pathway, can be observed in many cancer cells and tissues. There is evidence that the PI3K/Akt pathway is related to the drug resistance of NSCLC, which affects the sensitivity of lung cancer cells to cisplatin [18]. It has also been reported that downregulation of P-gp expression via inhibition of the $\mathrm{PI} 3 \mathrm{~K} / \mathrm{Akt} / \mathrm{mTOR}$ signaling pathway can induce apoptosis in multidrug-resistant cells $[19,20]$. According to our previous work, Ad-VT regulates P-gp protein levels by affecting the PI3K/Akt/mTOR signaling pathway. We used Ad-VT (MOI of 80 ) to infect cells and then detected the expression 
a

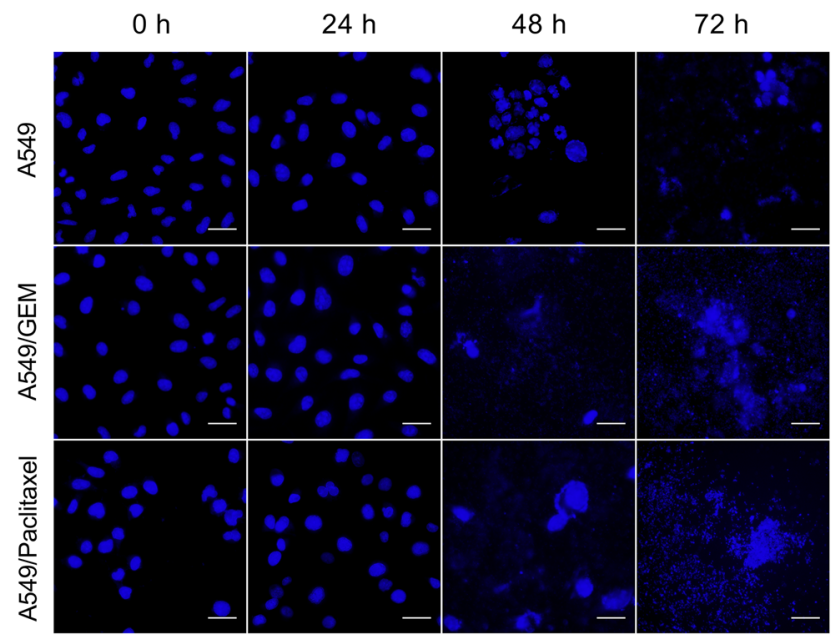

d
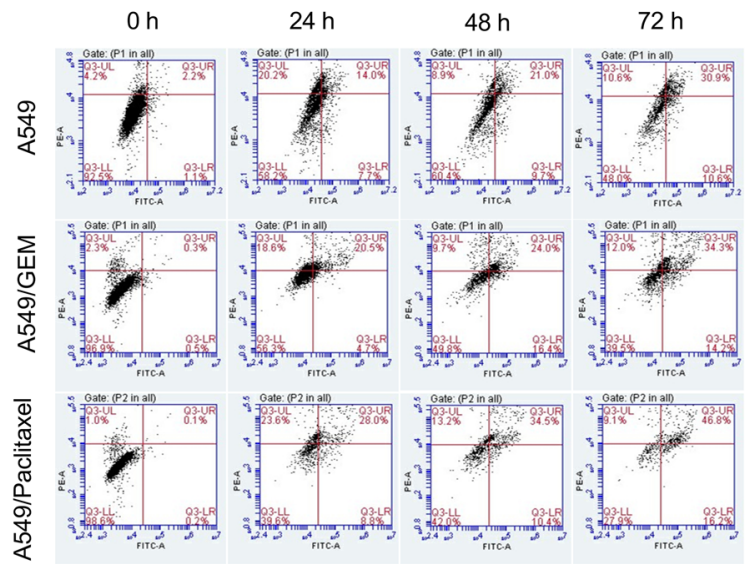

e

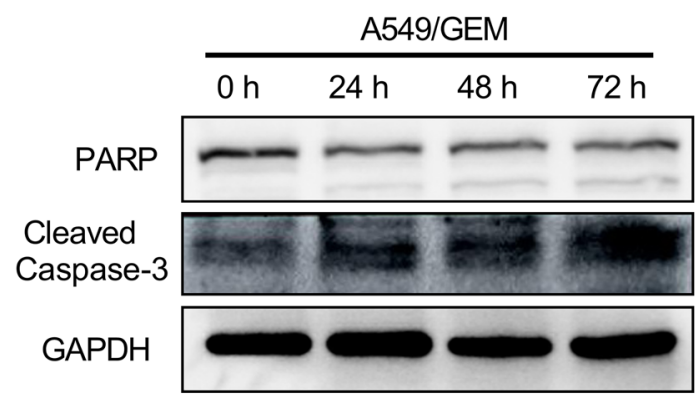

Fig. 3 Ad-VT treatment induces apoptosis in lung cells. a Cells were treated with Ad-VT at an MOI of 80. After 24, 48 and $72 \mathrm{~h}$, apoptotic cells were detected by Hoechst 33342 staining and observed under a fluorescence microscope. b-c Cells were treated with Ad-VT for $48 \mathrm{~h}$, and apoptotic cells were detected by Annexin V-FITC staining (b) and TUNEL staining (c) under a fluorescence microscope. d Cells were incubated in the presence or absence of Ad-VT at an MOI of 80 for $0,24,48$, and $72 \mathrm{~h}$, and apoptosis was determined by flow cytom- b

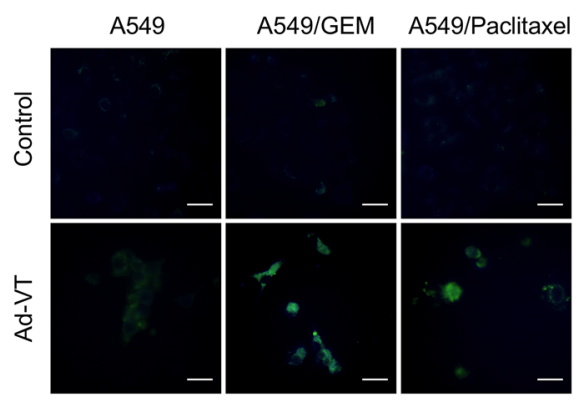

C
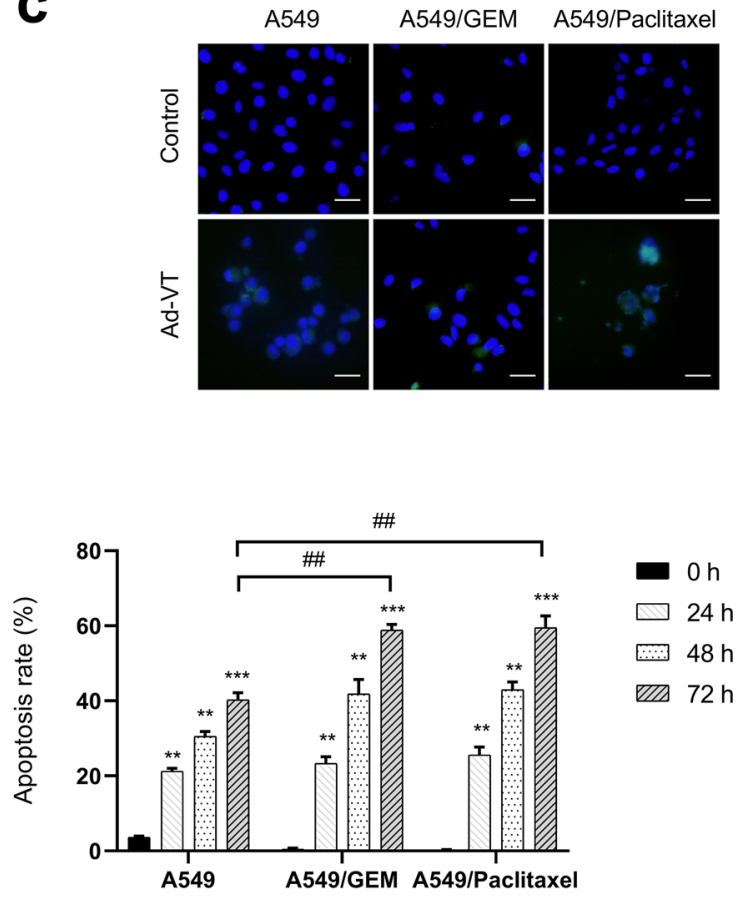

f

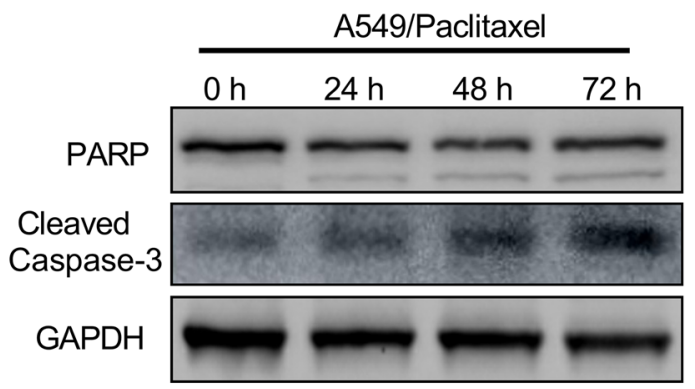

etry with Annexin V-FITC/PI staining. e-f The molecular changes related to apoptosis in A549/GEM and A549/Paclitaxel cells were detected by Western blot with anti-PARP and anti-cleaved-caspase 3 antibodies. The values represent the means $\pm \mathrm{SD}(\mathrm{n}=3) .{ }^{*} p<0.05$, $* * p<0.01$, *** $p<0.001$ compared with controls; \#\# $p<0.01$ compared with the corresponding group. Magnification, $200 \times$. Scale bars: $50 \mu \mathrm{m}$ 
a

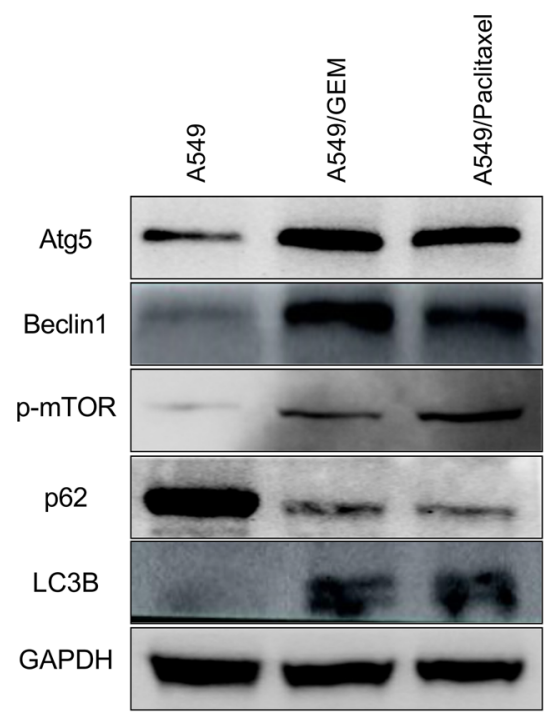

C

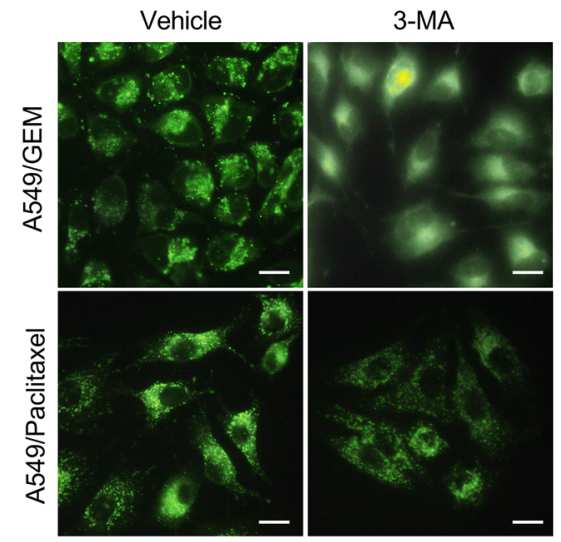

b
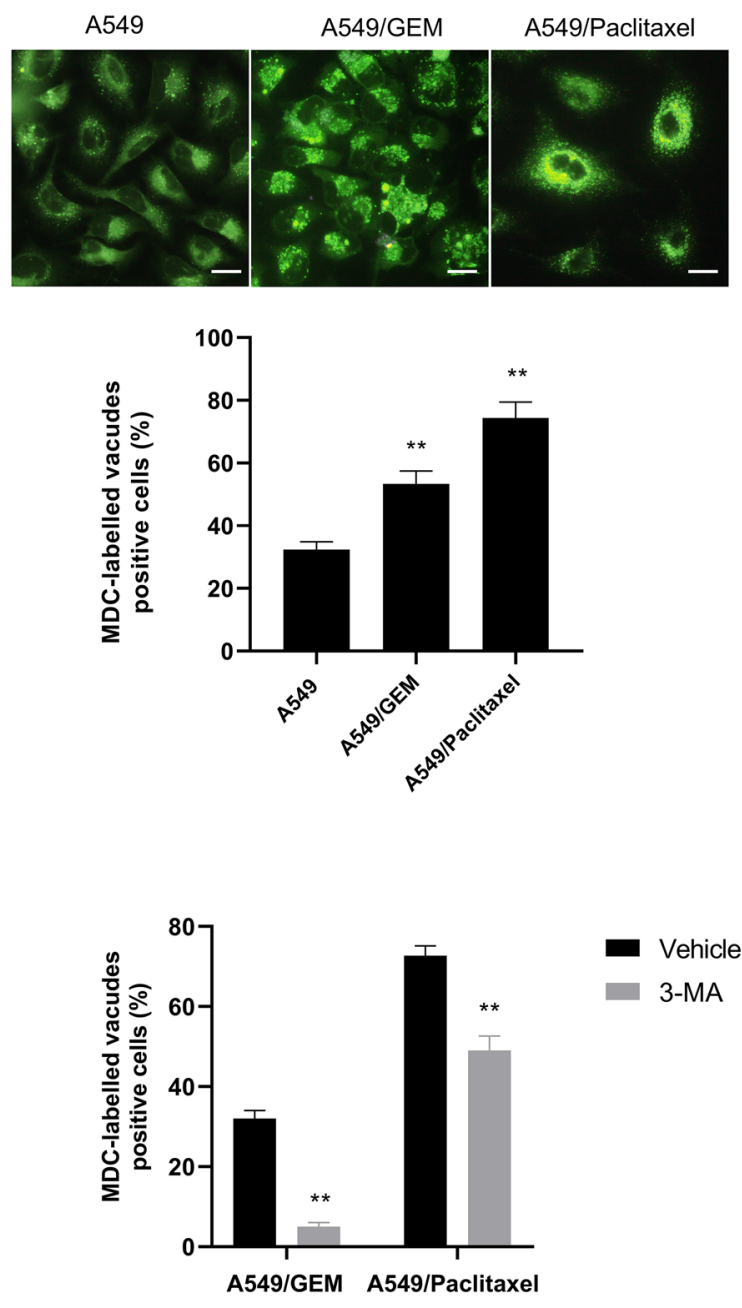

f

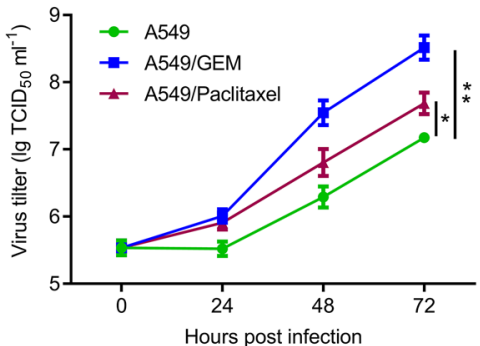

e

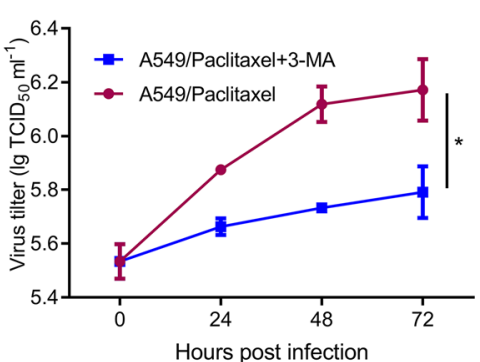

Fig. 4 High levels of basal autophagy in drug-resistant cell lines enhanced the antitumor effect of Ad-VT. a The expression of the autophagy-related proteins ATG5, Beclin1, p-mTOR, p62 and LC3-II in lung cancer cells was evaluated by Western blotting. b Autophagic vacuoles in lung cancer cells were identified by MDC staining. Scale bars: $25 \mu \mathrm{m}$. c After A549/GEM and A549/Paclitaxel cells were treated with 3-MA, autophagic vacuoles were detected by
MDC staining. d Detection of viral replication in A549, A549/GEM and A549/Paclitaxel lung cancer cells infected with Ad-VT at different time points. e-f After 3-MA treatment, viral replication in A549/ GEM and A549/Paclitaxel cells was detected at different time points after Ad-VT (MOI of 80 ) infection, $\mathrm{n}=8$ for each group. The data are presented as the means $\pm \mathrm{SD}, * p<0.05, * * p<0.01$ compared with the controls 
a

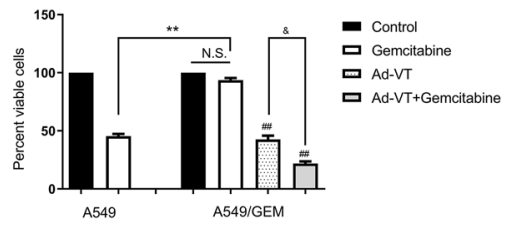

b

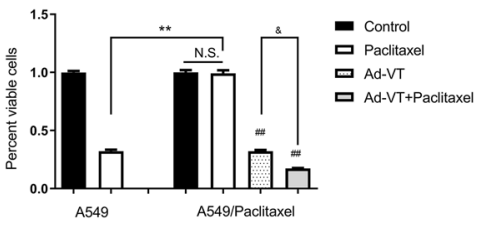

C
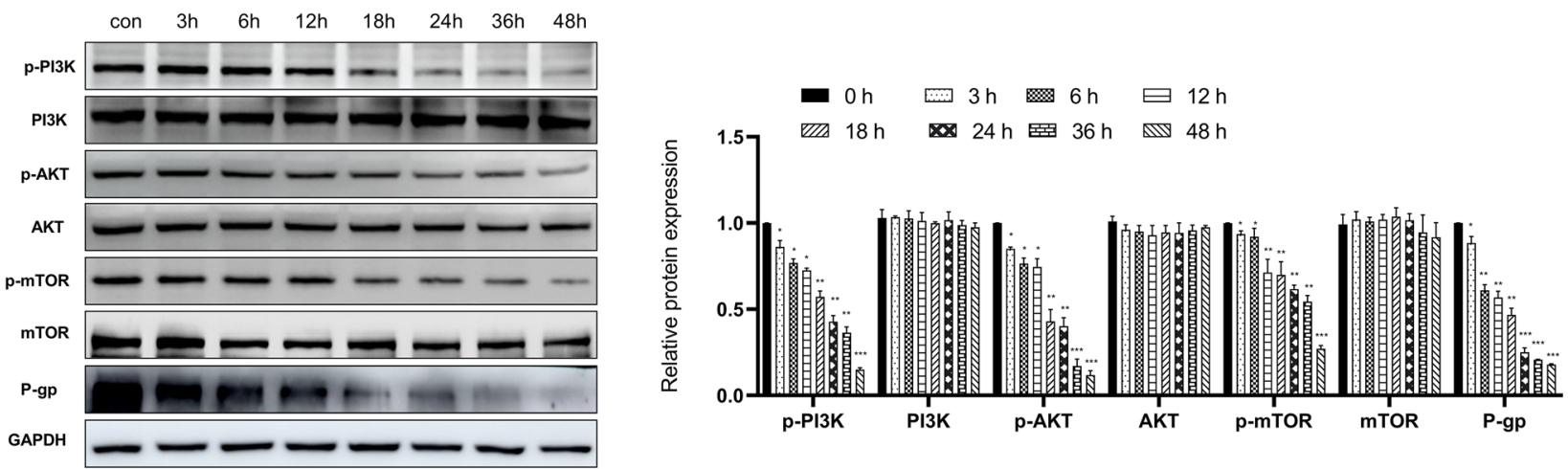

d
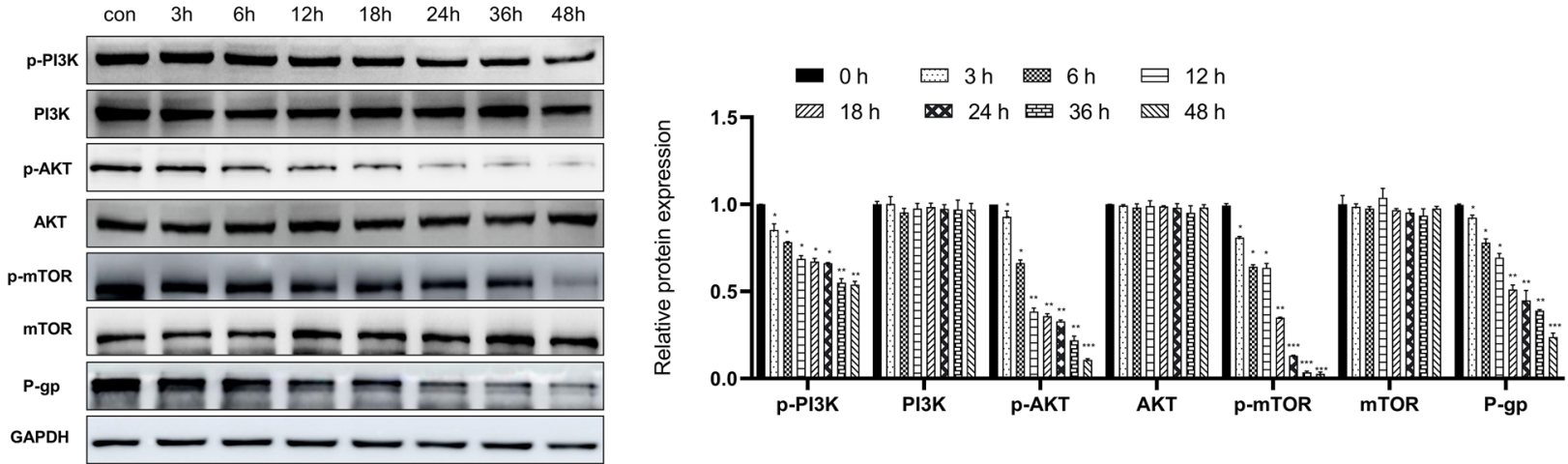

e

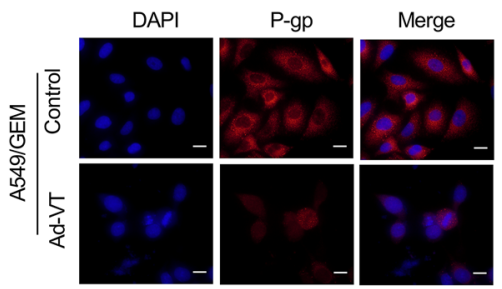

f

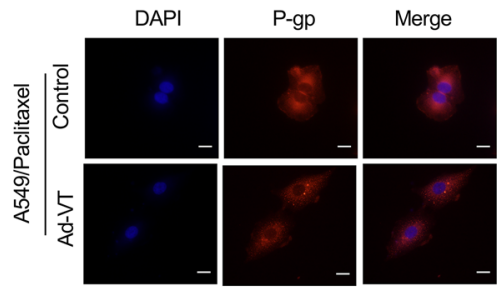

Fig. 5 Ad-VT combined with gemcitabine or paclitaxel can inhibit the growth of A549/GEM and A549/Paclitaxel cells and reduce their drug resistance. a-b A549 cells were treated with Ad-VT (MOI of 80); A549/GEM cells were treated with gemcitabine $(10 \mu \mathrm{g} / \mathrm{ml})$ and Ad-VT (MOI of 80) either alone or in combination; and A549/Paclitaxel cells were treated with paclitaxel $(10 \mu \mathrm{g} / \mathrm{ml})$ and Ad-VT (MOI of 80) either alone or in combination. All treatments lasted $48 \mathrm{~h}$, and viability was examined by the CCK- 8 assay. c-d Western blotting was used to analyze the expression levels of p-PI3k, PI3K, p-mTOR, mTOR, p-Akt, Akt and P-gp in A549/GEM and A549/Paclitaxel cells

treated with Ad-VT (MOI of 80) at different time points. All data are shown as the means $\pm \mathrm{SD}, \mathrm{n}=3$. e-f Immunofluorescence analysis of P-gp protein expression (red fluorescence) and the nucleus (blue fluorescence, DAPI staining) in A549/GEM and A549/Paclitaxel cells treated with Ad-VT (MOI of 80) for $24 \mathrm{~h}$. Scale bars: $25 \mu \mathrm{m}$. In untreated control cells, cell viability was considered to be $100 \%$. The data are presented as the means $\pm \mathrm{SD}, * p<0.05, * * p<0.01$ compared with controls. ${ }^{\#} p<0.05,{ }^{\# \#} p<0.01$ compared with the corresponding group. ${ }^{\&} p<0.05$ compared with the indicated group

of related proteins at different times after infection $(0,3,6$, 12, 18, 24, 36 and 48 h) (Fig. 5c, d). The Western blotting results showed that Ad-VT significantly inhibited p-PI3K,

p-Akt, p-mTOR and P-gp levels in a time-dependent manner. The immunofluorescence results revealed that Ad-VT induced the downregulation of $\mathrm{P}-\mathrm{gp}$ protein expression in 
A549/GEM and A549/Paclitaxel cells (Fig. 5e, f) consistent with the Western blot results. In conclusion, Ad-VT downregulates P-gp by reducing the expression of key proteins in the PI3K/Akt/mTOR signaling pathway, thereby interfering with the resistance of A549/GEM cells to gemcitabine or A549/Paclitaxel cells to paclitaxel.

\section{Ad-VT inhibited tumor growth in vivo}

To further study the antitumor effects of Ad-VT, gemcitabine and paclitaxel, a xenograft model was established in nude mice. Compared with the control treatment, Ad-VT, gemcitabine and paclitaxel treatment resulted in a significant decrease in tumor volume (Fig. 6a, b), suggesting that Ad-VT, gemcitabine and paclitaxel have inhibitory effects on lung cancer. IHC showed that compared with the PBS control group, the Ad-VT, gemcitabine and paclitaxel treatment groups had decreased Ki-67 expression in lung cancer tissues and increased TUNEL staining (Fig. 6c). By IHC, we also found that the protein expression of P-gp in the Ad-VT, gemcitabine or paclitaxel groups was lower than that in the PBS group (Fig. 6c). These results indicate that Ad-VT, gemcitabine and paclitaxel can inhibit the proliferation of tumor cells, promote apoptosis, and inhibit P-gp expression in vivo.

\section{Ad-VT increased the sensitivity of chemoresistant xenografts to gemcitabine and paclitaxel in a BALB/c nude mouse model}

To further study the therapeutic effect of Ad-VT combined with gemcitabine or paclitaxel, we established a xenograft tumor model in nude mice. Figure 7a illustrates the effects of the combination of Ad-VT and gemcitabine on the growth of A549/GEM-derived tumors. The results indicate that compared with the control group, the gemcitabine-treatment group had only slightly smaller tumors, indicating that gemcitabine has a less pronounced effect on drug-resistant tumors. The tumor volume of the Ad-VT group was significantly smaller than that of the control group, and there a significant difference on day 30; these data indicated that Ad-VT was a significant inhibitory effect on the growth of drug-resistant tumors $(p<0.05)$. As expected, the tumor volume of the Ad-VT plus gemcitabine group grew more slowly and was significantly smaller than that of the control, gemcitabine and Ad-VT groups, demonstrating that the combination therapy had a significant inhibitory effect on tumor growth $(p<0.05)$. After combined treatment, the survival time of mice was significantly prolonged. The combination of Ad-VT and gemcitabine improved the survival rate and prolonged the survival time of mice (Fig. 7b).

The synergistic effect of Ad-VT and gemcitabine was further confirmed by histological assessments of tumors in each group (Fig. 7c). The H\&E staining results showed that in the Ad-VT combined with gemcitabine group, there was nucleolysis, pyknosis and an increased number of cytoplasmic vacuoles, and the inhibition of tumor cell proliferation was the most obvious. The protein expression of P-gp in the Ad-VT combined with gemcitabine group was significantly lower than that in either monotherapy group or the control group $(p<0.01)$. The TUNEL results revealed that Ad-VT combined with gemcitabine treatment was significantly more effective as an antitumor therapy than either monotherapy group or the control group. Assessment of Ki-67 expression showed that Ad-VT combined with gemcitabine treatment resulted in significantly lower Ki-67 expression than did either monotherapy and control treatment. These results consistently indicate that Ad-VT combined with gemcitabine has the strongest antitumor activity against A549/ GEM xenografts in nude mice compared with that of the control treatment.

Next, we further conducted the same experiments described above with Ad-VT combined with paclitaxel in A549/Paclitaxel cell-derived xenografts. As shown in Fig. 7d, e and f, as expected, the tumor volume of the Ad-VT combined with paclitaxel group on days 20,25 , and 30 was smaller than that of either monotherapy group or the control group, with a statistically significant difference observed on day 56. The survival rate at day 56 was $10 \%$ in the control group, $10 \%$ in the paclitaxel group, $30 \%$ in the Ad-VT group, and $60 \%$ in the combination treatment group. These results showed that the survival rate of tumor-bearing mice in the combined treatment group was significantly higher than that in the monotherapy groups and the control group. The H\&E results showed that nucleolysis and the number of cytoplasmic vacuoles were increased in the combination treatment group (Fig. 7f). The TUNEL results indicated that Ad-VT combined with paclitaxel was significantly stronger than the monotherapy groups and the control group in inducing cell death. The Ki-67 results revealed that tumor proliferation in the Ad-VT combined with paclitaxel group was significantly lower than that in the Ad-VT alone and control groups. Moreover, Ad-VT combined with paclitaxel significantly reduced the protein expression of P-gp. Compared with the control treatment, Ad-VT combined with paclitaxel had the most effective antitumor activity in the A549/Paclitaxel xenograft tumor model in nude mice.

\section{Discussion}

Chemoresistance has always been a major obstacle to the success of tumor chemotherapy. Although gemcitabine and paclitaxel, as first-line antitumor drugs, have been widely used in the treatment of NSCLC, chemotherapy failure is often caused by the development of drug resistance. To 


\section{a}
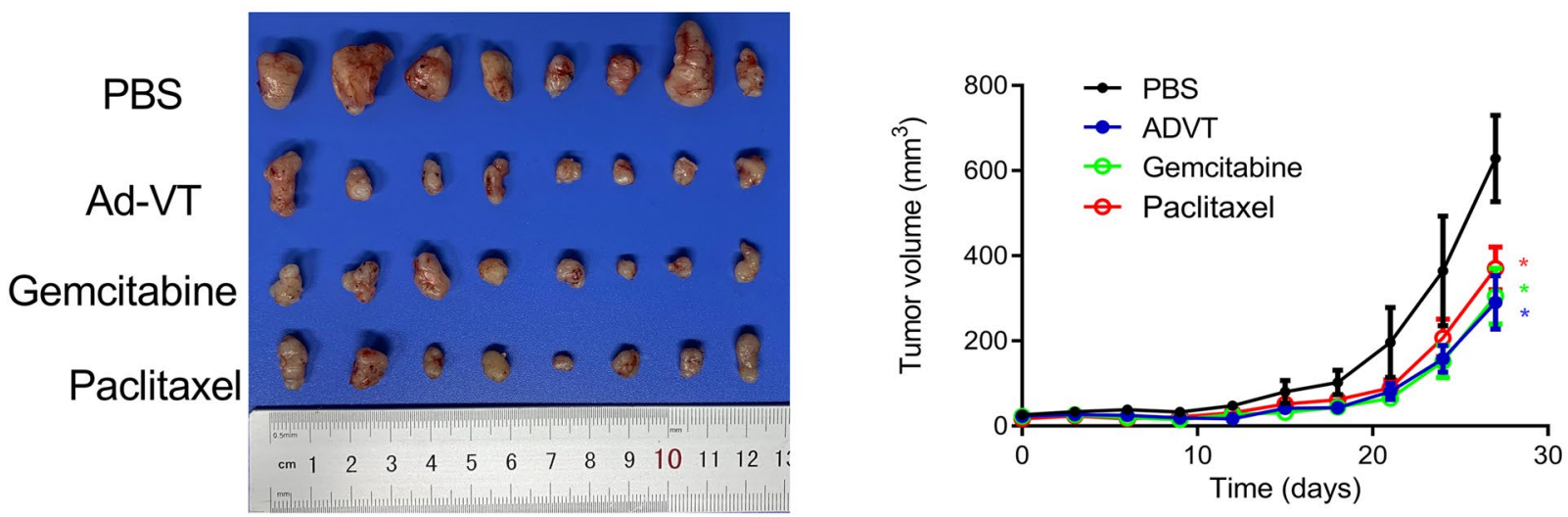

C

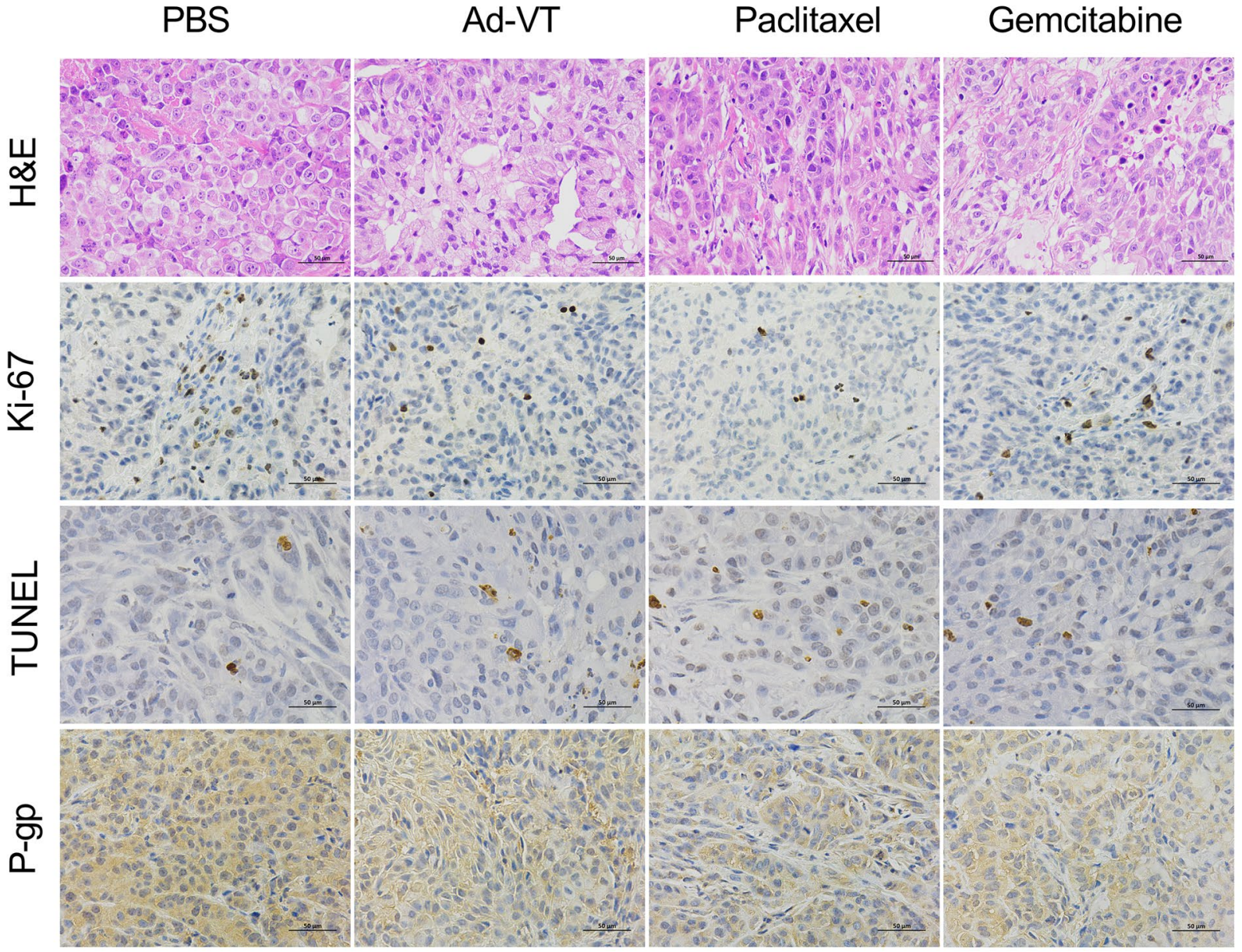

Fig. 6 Effect of Ad-VT, gemcitabine and paclitaxel on A549 cellderived xenografts in a BALB/c nude mouse model. a Xenograft tumors from nude mice treated with Ad-VT, gemcitabine and paclitaxel were excised, imaged and measured. b The tumor volume curves of mice treated with gemcitabine $(10 \mathrm{mg} / \mathrm{kg})$, paclitaxel $(10 \mathrm{mg} / \mathrm{kg})$, Ad-VT $\left(1 \times 10^{9}\right.$ plaque-forming units $)$ and PBS, $\mathrm{n}=10$ / group, $* p<0.05$. c The histological features of sections from A549 cell-derived tumors were characterized by H\&E staining. Ki-67 and P-gp expression in the xenografts was detected by immunohistochemical staining, and TUNEL staining was performed. Images are typical of 3 independent assays. Magnification, $400 \times$. Scale bars: $50 \mu \mathrm{m}$ 
Fig. 7 The synergistic effect of Ad-VT with gemcitabine or paclitaxel on A549/GEM- and A549/Paclitaxel-derived xenografts in a BALB/c nude mouse model. a, d Growth curve of tumor volumes in the different treatment groups. Each data point indicates the mean \pm SD. $\mathrm{n}=7, * p<0.05, * * p<0.01$ vs. the PBS group. b, e The survival rate of mice in the different treatment groups. $\mathbf{c}, \mathbf{f}$ The histological features of subcutaneous tumors derived from A549/GEM and A549/Paclitaxel cells were characterized by H\&E staining. Ki-67 and P-gp expression in the xenografts was detected by immunohistochemical staining, and TUNEL staining was performed. Images are typical of 3 independent assays. Magnification, $400 \times$. Scale bars: $50 \mu \mathrm{m}$ a

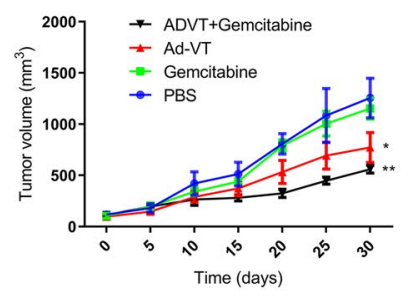

C

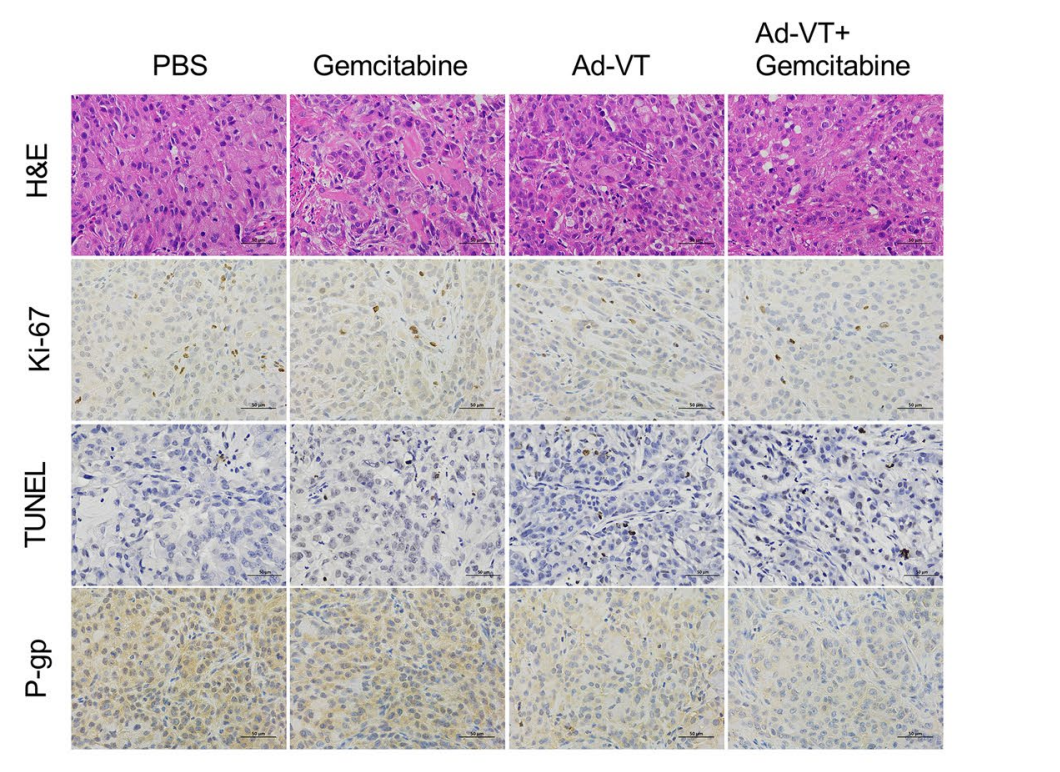

d

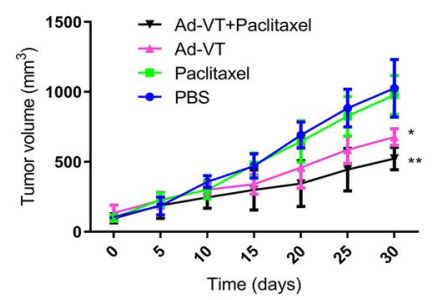

e

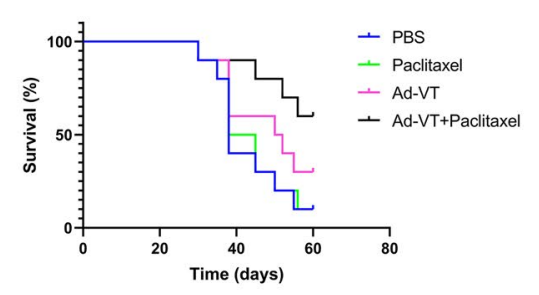

f

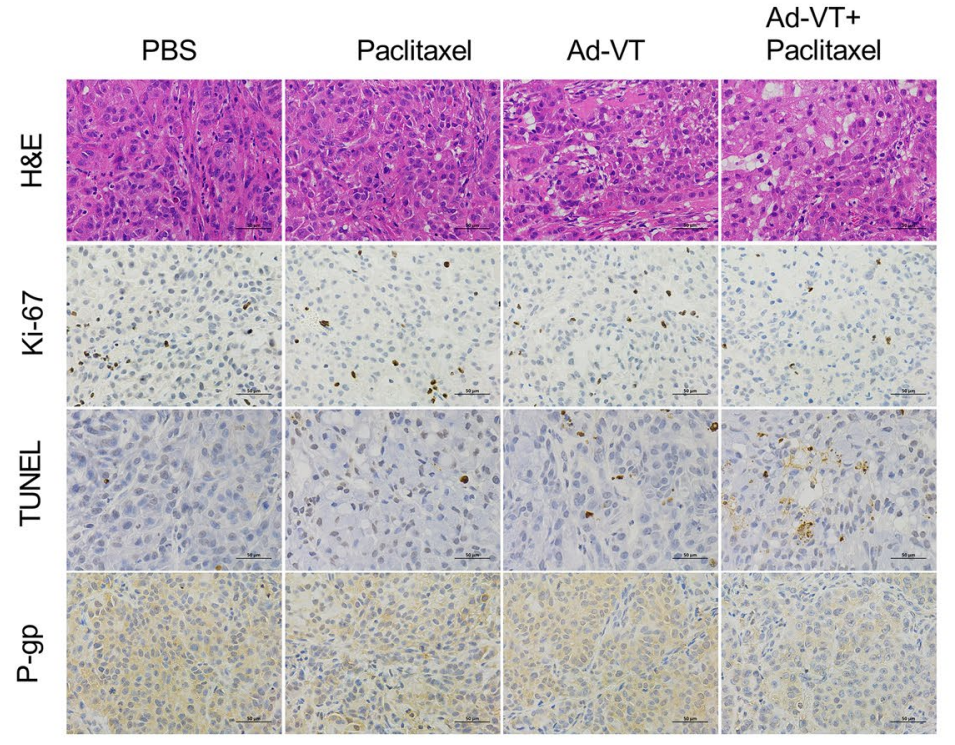


Table 1 The resistance index (RI) of various compounds in A549/GEM and A549/ Paclitaxel cells (mean \pm SD, $\mathrm{n}=3$ )

\begin{tabular}{lllll}
\hline Compounds & IC 50 $(\mathrm{nm})$ & & \multirow{2}{*}{ RI } \\
\cline { 2 - 4 } & A549 & A549/GEM & A549/Paclitaxel & \\
\hline Paclitaxel & $31.44 \pm 1.72$ & $20.22 \pm 1.480$ & $3193 \pm 207.6$ & A549/GEM:1 \\
& & & & A549/Paclitaxel:103 \\
Gemcitabine & $481.3 \pm 88.35$ & $8369 \pm 474.6$ & $406.2 \pm 87.11$ & A549/GEM:17 \\
& & & & A549/Paclitaxel:1 \\
\hline
\end{tabular}

overcome MDR, a large number of studies have been carried out to elucidate the complex mechanism(s) involved. The high expression of multidrug resistance gene 1 (MDR1) in tumor tissue has been reported to be related to poor prognosis and MDR in tumor patients [21].

$\mathrm{P}$-gp mediates MDR, and P-gp overexpression in drugresistant tumor cells is one of the driving factors of tumor resistance [22]. High and persistent expression of P-gp increases the resistance of the lung squamous cell carcinoma cell line $\mathrm{CH} 27$ to doxorubicin [22]. At present, many drugs have been developed and applied to prevent or mitigate chemotherapy resistance in lung cancer, including calcium channel blockers, antiarrhythmic drugs and some components of traditional Chinese medicine [23]. Although there are drugs that can temporarily increase the concentration of chemotherapeutic drugs to sufficient levels that will kill tumor cells, they cannot be widely used in the clinic due to their instability, nonspecificity and adverse effects. Therefore, finding effective drugs or treatment strategies to inhibit or reverse MDR is the key to improving the survival of lung cancer patients.

With advances in molecular biology and gene engineering technologies, many novel treatment schemes have been developed, including gene targeting and OV therapy. Compared with other cancer treatment methods, OVs have a broad antitumor spectrum, are safe and reliable, are less toxic with fewer adverse effects, and exert synergistic effects with other anticancer drugs [24]. Therefore, the development and clinical application of adenoviruses as drug vectors have been widely studied, and progress has been made. Adenovirusbased tumor-targeted therapy is predicted to replace traditional tumor therapy. In the present study, we investigated the effects of Ad-VT (Ad-hTERTp-E1a-Apoptin), a new recombinant $\mathrm{OV}$ that can specifically replicate in tumor cells and express apoptin protein efficiently (thus playing an effective role in tumor cell death) [25], on gemcitabine-resistant and paclitaxel-resistant lung cancer cells in vitro and on their corresponding xenograft tumor models in vivo. Ad-VT was observed to enhance the chemosensitivity of drug-resistant cells in vitro and in vivo. Our previous studies have shown that Ad-VT can inhibit a variety of tumor cells and has good safety [26]. The tumor-specific promoter hTERTp can activate the replication and/or expression of certain genes in tumor cells and is located upstream of the adenovirus promoter E1a gene and apoptin gene in Ad-VT. This recombinant design bestows Ad-VT with specific replication and killing abilities.

In the present study, we found that Ad-VT has a high replication rate in drug-resistant lung cancer cells and can not only induce apoptosis of drug-resistant lung cancer cells but also reduce the expression of the P-gp protein. It is speculated that Ad-VT is a promising drug for the treatment of gemcitabine- or paclitaxel-resistant NSCLC, with previous studies reporting that persistent elevated P-gp expression increases the resistance of DOX-resistant $\mathrm{CH} 27$ cells to DOX [27]. Our Western blot results showed that P-gp expression in A549/GEM and A549/Paclitaxel cells was higher than that in parental A549 cells (Fig. 1a), which was consistent with the drug resistance characteristics of A549/ GEM and A549/Paclitaxel cells.

The Kaplan-Meier Plotter database showed that P-gp expression in lung cancer tissues was significantly higher than that in normal lung tissues (Fig. 1b). It is generally believed that the ability to induce apoptosis is one of the most basic elements in the development of antitumor drugs [28], and apoptosis activation is related to the cytotoxic effect of chemotherapy on tumor cells. In this study, our results show that Ad-VT not only inhibits proliferation but also induces apoptosis in A549, A549/GEM and A549/ Paclitaxel cells in a dose- and time-dependent manner; furthermore, the rates of proliferation inhibition and apoptosis induction were higher in A549/GEM and A549/Paclitaxel cells than in A549 cells.

An increasing number of studies have indicated that autophagy plays an important role in tumor drug resistance. Autophagy is an evolutionarily conserved process that provides metabolic support for the degradation and renewal of long-lived proteins and dysfunctional organelles to maintain cell homeostasis. Autophagy occurs continuously at basal metabolic levels in normal cells and is upregulated in response to stressors such as starvation, oxidative stress or cytotoxic drug therapy [29]. It has also been reported that tumor cells can induce chemotherapy resistance via autophagy [30-32]. LC3-II is a reliable marker for monitoring autophagy and autophagy-related processes in mammals [33, 34], and increased levels of p62 (sqstm1) indicate that autophagy is blocked. When LC3-II is upregulated, p62 is downregulated, indicating that autophagy is active, 
whereas decreased LC3-II and increased p62 levels indicate that autophagy is inhibited [28]. mTOR is an evolutionarily conserved serine/threonine kinase that regulates autophagy [35]. ATG5 accumulation leads to negative feedback to signals upstream of mTOR, resulting in reductions in the level of phosphorylated mTOR and consequent activation of the autophagic pathway [36]. Our results showed that compared with A549 cells, A549/GEM and A549/Paclitaxel cells had increased expression levels of Atg5, p-mTOR and LC3-II and decreased expression of p62 (Fig. 4a). These data are in agreement with the literature stating that autophagy is enhanced in drug-resistant cell lines. In addition, MDClabeled vacuole analysis revealed that A549/GEM and A549/Paclitaxel cells had stronger basal autophagy activity than parental A549 cells. This result indicated that resistance to gemcitabine and paclitaxel was related to enhanced autophagy that is, autophagy is a survival mechanism of gemcitabine- or paclitaxel-resistant cell lines against chemotherapy drugs. Research shows that the autophagy inducer rapamycin improves the oncolytic efficacy of Newcastle disease OVs in drug-resistant lung cancer cells through enhanced autophagy [33]. The results of the virus titer assay showed that Ad-VT had a stronger replication ability in A549/GEM and A549/Paclitaxel cell lines than in A549 cells (Fig. 4d). The autophagy inhibitor 3-MA decreased the level of autophagy in a drug-resistant lung cancer cell line and weakened the replication ability of Ad-VT, thus affecting the cytotoxicity of Ad-VT (Fig. 4d, e and f). Combined with the above results, these data suggest that autophagy contributes to the oncolytic effect of Ad-VT in drug-resistant lung cancer cell lines.

Increasingly, studies have shown that the PI3K/Akt/ mTOR signaling pathway is related to tumor drug resistance [37-39]. Akt inhibitors increase the sensitivity of cancer cells to chemotherapy and radiotherapy [40, 41], and inhibitors of PI3K and mTOR play an important role in the treatment and drug resistance of cancer [41]. Some studies have reported that resveratrol can downregulate P-gp expression by inhibiting the PI3K/Akt/mTOR signaling pathway and subsequently reduce the drug resistance of cancer cells [19, 20], suggesting that the PI3K/Akt/mTOR signaling pathway is closely related to drug resistance. This conclusion is logical, as P-gp (a transmembrane drug transporter) is involved in drug resistance and is regulated by the $\mathrm{PI} 3 \mathrm{~K} / \mathrm{Akt} / \mathrm{mTOR}$ signaling pathway. Our results showed that Ad-VT downregulated the expression levels of not only p-pI3k, p-Akt and p-mTOR pathway proteins but also P-gp protein in chemoresistant A549/GEM and A549/Paclitaxel cancer cell lines (Fig. 5c), resulting in decreased PI3K/Akt/mTOR signaling pathway function and reduced chemotherapy resistance of drug-resistant lung cancer cell lines. These data suggest that Ad-VT plays a role in chemotherapy resistance at the molecular level by affecting the protein expression of constituents of the PI3K/Akt/mTOR signaling pathway, which can be exploited to improve the sensitivity of drug-resistant lung cancer cell lines to chemotherapy.

The nude mouse xenograft tumor model was established with A549, A549/GEM and A549/Paclitaxel cells. We observed that in xenografts derived from A549 cells, the tumor volumes of the Ad-VT, gemcitabine, and paclitaxel groups were significantly reduced compared with those of the control group (Fig. 6a, b), with IHC showing that Ki-67 and P-gp expression was decreased and TUNEL staining was increased (Fig. 6c). In xenografts derived from A549/GEM cells, Ad-VT combined with gemcitabine caused significant tumor growth inhibition, which was significantly stronger than that caused by either gemcitabine or Ad-VT alone and by the control treatment. Moreover, compared with those from the monotherapy and control groups, the xenografts from the Ad-VT and combination gemcitabine group exhibited significant nucleolysis, increased number of vacuoles, decreased Ki-67 expression, increased TUNEL staining, and decreased P-gp expression. In xenografts derived from A549/Paclitaxel cells, the results were similar to those of A549/GEM xenograft tumors. In conclusion, Ad-VT alone or in combination with gemcitabine or paclitaxel had obvious antitumor effects in a chemoresistant tumor model. More importantly, Ad-VT can improve the chemosensitivity of chemoresistant lung cancer cells in vivo. The inhibitory effect of gemcitabine and paclitaxel on A549/GEM and A549/Paclitaxel cells, respectively, was significantly enhanced when combined with Ad-VT, indicating that these combinations exhibit a synergistic anticancer effect, suppress the drug resistance to chemotherapy drugs, and reduce the side effects of gemcitabine and paclitaxel. Therefore, in future research and clinical applications, the effectiveness, safety and optimization of the administration of chemotherapeutic drugs in combination with Ad-VT shoud be the main goals to better develop and utilize the application of Ad-VT in drug-resistant tumors.

\section{Conclusions}

Ad-VT can significantly inhibit the proliferation of gemcitabine- and paclitaxel-resistant lung cancer cells mainly by inducing apoptosis. Ad-VT can also improve the sensitivity of drug-resistant lung cancer cells to chemotherapy drugs. This mechanism can be divided into two aspects: first, the replication of Ad-VT increases because of the strong autophagy activity of drug-resistant cells; and second, Ad-VT mainly reduces the chemotherapy resistance of drug-resistant lung cancer cells by inhibiting PI3K/Akt/mTOR pathway function and downregulating P-gp expression. Therefore, 
our study suggests that the combination of Ad-VT and chemotherapeutic drugs is a promising therapeutic strategy to overcome chemoresistance.

Acknowledgements We would like to thank the contributors to The Human Protein Atlas (Kaplan-Meier Plotter) online database for the available data.

Authors' contributions Gaojie Song, Jinbo Fang and Ningyi Jin designed the study. Gaojie Song, Jinbo Fang, Chao Shang, Yiquan Li, Lili Sun, Zhiru Xiu, Zirui Liu, Yaru Li, Xia Yang and Chengcheng Ge participated in the tissue sample selection and experiments. Gaojie Song, Jinbo Fang and Ningyi Jin performed the experiments and analyzed the data. Gaojie Song and Jinbo Fang wrote the manuscript. Ningyi Jin, Xiao Li and Jinbo Fang revised the manuscript. Jinbo Fang provided funding support. All authors read and approved the final manuscript.

Funding This work was supported by the Natural Science Foundation of Jilin Province (Grant 20210101046JC).

Data availability The datasets generated and/or analyzed during the current study are available from the corresponding author on reasonable request.

\section{Declarations}

Ethics approval and consent to participate All animal experimental protocols were approved by the Institutional Animal Care and Use Committee (IACUC) of the Changchun Veterinary Research Institute, Chinese Academy of Agricultural Sciences (Changchun, China), and all efforts were made to minimize animal suffering and reduce the number of animals used for the experiments.

Informed consent Informed consent was obtained from all individual participants included in the study.

\section{Consent for publication Not applicable.}

Research involving human participants and/or animals The animal experimental protocols were approved by the IACUC of the Changchun Veterinary Research Institute, Chinese Academy of Agricultural Sciences (Changchun, China) (10ZDGG007). The manuscript does not contain human clinical studies or patient data.

Conflict of interests The authors declare that they have no competing interests.

Open Access This article is licensed under a Creative Commons Attribution 4.0 International License, which permits use, sharing, adaptation, distribution and reproduction in any medium or format, as long as you give appropriate credit to the original author(s) and the source, provide a link to the Creative Commons licence, and indicate if changes were made. The images or other third party material in this article are included in the article's Creative Commons licence, unless indicated otherwise in a credit line to the material. If material is not included in the article's Creative Commons licence and your intended use is not permitted by statutory regulation or exceeds the permitted use, you will need to obtain permission directly from the copyright holder. To view a copy of this licence, visit http://creativecommons.org/licenses/by/4.0/.

\section{References}

1. Hu SS, Yuan YL, Song ZZ et al (2018) Expression Profiles of microRNAs in Drug-Resistant Non-Small Cell Lung Cancer Cell Lines Using microRNA Sequencing. Cell Physiol Biochem 51:2509-2522. https://doi.org/10.1159/000495921

2. Hasanovic A, Mus-Veteau I (2018) Targeting the Multidrug Transporter Ptch1 Potentiates Chemotherapy Efficiency. Cells 7:undefined. https://doi.org/10.3390/cells70801073

3. Choi $\mathrm{CH}$ (2005) ABC transporters as multidrug resistance mechanisms and the development of chemosensitizers for their reversal. Cancer Cell Int 5:30. https://doi.org/10.1186/ 1475-2867-5-30

4. Cook M, Chauhan A (2020) Clinical Application of Oncolytic Viruses: A Systematic Review. Int J Mol Sci 21:undefined. https:// doi.org/10.3390/ijms21207505

5. Zhang Q, Liu F (2020) Advances and potential pitfalls of oncolytic viruses expressing immunomodulatory transgene therapy for malignant gliomas. Cell Death Dis 11:485. https://doi.org/10. 1038/s41419-020-2696-5

6. Shi T, Song XR, Wang Y et al (2020) Combining Oncolytic Viruses With Cancer Immunotherapy: Establishing a New Generation of Cancer Treatment. Front Immunol 11:683. https://doi. org/10.3389/fimmu.2020.00683

7. Lawler SE, Speranza MC, Cho CF et al (2017) Oncolytic Viruses in Cancer Treatment: A Review. J Hematol Oncol 3:841-849. https://doi.org/10.1001/jamaoncol.2016.2064

8. Fang JB, Zhu GZ, Zhu YL et al (2020) Antitumor effects of apoptin expressed by the dual cancer-specific oncolytic adenovirus - a review. Eur Rev Med Pharmacol Sci 24:11334-11343. https://doi. org/10.26355/eurrev_202011_23624

9. Li X, Liu Y, Wen ZM et al (2010) Potent anti-tumor effects of a dual specific oncolytic adenovirus expressing apoptin in vitro and in vivo. Mol Cancer 9:10. https://doi.org/10.1186/1476-4598-9-10

10. Pan B, Yang LF, Wang J et al (2014) C-Abl tyrosine kinase mediates neurotoxic prion peptide-induced neuronal apoptosis via regulating mitochondrial homeostasis. Mol Neurobiol 49:1102-1116. https://doi.org/10.1007/s12035-014-8646-4

11. Pan B, Zhang HM, Cui TX et al (2017) TFEB activation protects against cardiac proteotoxicity via increasing autophagic flux. J Mol Cell Cardiol 113:51-62. https://doi.org/10.1016/j.yjmcc. 2017.10.003

12. Pan B, Li J, Parajuli N et al (2020) The Calcineurin-TFEB-p62 Pathway Mediates the Activation of Cardiac Macroautophagy by Proteasomal Malfunction. Circ Res 127:502-518. https://doi.org/ 10.1161/CIRCRESAHA.119.316007

13. Wang YS, Zhao DM, Pan B et al (2015) Death Receptor 6 and Caspase-6 Regulate Prion Peptide-Induced Axonal Degeneration in Rat Spinal Neurons. J Mol Neurosci 56:966-976. https://doi. org/10.1007/s12031-015-0562-1

14. Pan YQ, Wang S, Li P et al (2021) Apoptotic investigation of brain tissue cells in dogs naturally infected by canine distemper virus. Virol J 18(1):165. https://doi.org/10.1186/s12985-021-01635-8

15. Pan B, Lewno MT, Wu PL et al (2019) Highly Dynamic Changes in the Activity and Regulation of Macroautophagy in Hearts Subjected to Increased Proteotoxic Stress. Front Physiol 10:758. https://doi.org/10.3389/fphys.2019.00758

16. Zhao ZH, Li J, Jiang Y et al (2017) CLDN1 Increases Drug Resistance of Non-Small Cell Lung Cancer by Activating Autophagy via Up-Regulation of ULK1 Phosphorylation. Med Sci Monit 23:2906-2916. https://doi.org/10.12659/msm.904177

17. Biederbick A, Kern HF, Elsässer HP (1995) Monodansylcadaverine (MDC) is a specific in vivo marker for autophagic vacuoles. Eur J Cell Biol 66:3-14. https://doi.org/10.1089/dna.1995.14.87 
18. Shi H, Pu J, Zhou XL et al ( 2017) Silencing long non-coding RNA ROR improves sensitivity of non-small-cell lung cancer to cisplatin resistance by inhibiting PI3K/Akt/mTOR signaling pathway. Tumour Biol 39:undefined. https://doi.org/10.1177/ 1010428317697568

19. Wang L, Wang CY, Jia YM et al (2016) Resveratrol Increases Anti-Proliferative Activity of Bestatin Through Downregulating P-Glycoprotein Expression Via Inhibiting PI3K/Akt/mTOR Pathway in K562/ADR Cells. J Cell Biochem 117:1233-1239. https:// doi.org/10.1002/jcb. 25407

20. Song L, Zhou ZG, Gan YB et al (2019) Long noncoding RNA OIP5-AS1 causes cisplatin resistance in osteosarcoma through inducing the LPAAT $\beta / \mathrm{PI} 3 \mathrm{~K} / \mathrm{AKT} / \mathrm{mTOR}$ signaling pathway by sponging the miR-340-5p. J Cell Biochem 120:9656-9666. https://doi.org/10.1002/jcb.28244

21. Mo DL, Fang HB, Niu KF et al (2016) Human Helicase RECQL4 Drives Cisplatin Resistance in Gastric Cancer by Activating an AKT-YB1-MDR1 Signaling Pathway. Cancer Res 76:3057-3066. https://doi.org/10.1158/0008-5472.CAN-15-2361

22. Takara K, Sakaeda T, Kakumoto M et al (2009) Effects of $\alpha$-Adrenoceptor Antagonist Doxazosin on MDR1-Mediated Multidrug Resistance and Transcellular Transport. Oncol Res 17:527-533. https://doi.org/10.3727/096504009789745601

23. Xu MG, Tang XW, Guo JJ et al (2017) Reversal effect of adenovirusmediated human interleukin 24 transfection on the cisplatin resistance of A549/DDP lung cancer cells. Oncol Rep 38:2843-2851. https://doi.org/10.3892/or.2017.6002

24. Wang J, Li YQ, Li SZ et al (2020) Anti-tumor Synergistic Effect of a Dual Cancer-Specific Recombinant Adenovirus and Paclitaxel on Breast Cancer. Front Onco 10:244. https://doi.org/10. 3389/fonc.2020.00244

25. Zhang MC, Wang JH, Li C et al (2013) Potent growth-inhibitory effect of a dual cancer-specific oncolytic adenovirus expressing apoptin on prostate carcinoma. Int J Oncol 42:1052-1060. https:// doi.org/10.3892/ijo.2013.1783

26. Chen S, Li YQ, Yin XZ et al (2019) Recombinant adenoviruses expressing apoptin suppress the growth of MCF-7 breast cancer cells and affect cell autophagy. Oncol Rep 41:2818-2832. https:// doi.org/10.3892/or.2019.7077

27. Chen YL, Yang TY, Chen KC et al (2016) Hypoxia can impair doxorubicin resistance of non-small cell lung cancer cells by inhibiting MRP1 and P-gp expression and boosting the chemosensitizing effects of MRP1 and P-gp blockers. Cell Oncol (Dordr) 39:411-433. https://doi.org/10.1007/s13402-016-0285-5

28. Chen S, Wu DD, Sang XB et al (2017) The lncRNA HULC functions as an oncogene by targeting ATG7 and ITGB1 in epithelial ovarian carcinoma. Cell Death Dis 8:e3118. https://doi.org/10. 1038/cddis.2017.486

29. Ichimiya T, Yamakawa T, Hirano T et al (2020) Autophagy and Autophagy-Related Diseases: A Review. Int J Mol Sci 21:1-21. https://doi.org/10.3390/ijms21238974
30. Onorati AV, Dyczynski M, Ojha R et al (2018) Targeting autophagy in cancer. Cancer 124:3307-3318. https://doi.org/10. 1002/cncr.31335

31. Wu T, Liang X, Wang MC et al (2015) Autophagy facilitates lung adenocarcinoma resistance to cisplatin treatment by activation of AMPK/mTOR signaling pathway. Drug Des Devel Ther 9:6421-6431. https://doi.org/10.2147/DDDT.S95606

32. He J, Yu JJ, Xu Q et al (2015) Downregulation of ATG14 by EGR1-MIR152 sensitizes ovarian cancer cells to cisplatin-induced apoptosis by inhibiting cyto-protective autophagy. Autophagy 11:373-384. https://doi.org/10.1080/15548627.2015.1009781

33. Ke J, Li YC, Zhu QM et al (2014) Pharmacological modulation of autophagy enhances Newcastle disease virus-mediated oncolysis in drug-resistant lung cancer cells. BMC Cancer 14:551. https:// doi.org/10.1186/1471-2407-14-551

34. Zhang H, Chang JT, Guo B et al (2015) Guidelines for monitoring autophagy in Caenorhabditis elegans. Autophagy 11:9-27. https:// doi.org/10.1080/15548627.2014.1003478

35. Kim YC, Guan KL (2015) mTOR: a pharmacologic target for autophagy regulation. J Clin Invest 125:25-32. https://doi.org/10. 1172/JCI73939

36. Jiang SY, Chang H, Deng SJ et al (2019) Icariin enhances the chemosensitivity of cisplatinresistant ovarian cancer cells by suppressing autophagy via activation of the AKT/mTOR/ATG5 pathway. Int J Oncol 54:1933-1942. https://doi.org/10.3892/ijo. 2019.4785

37. Zhou DY, Liu WJ, Liang SH et al (2018) Apoptin-derived peptide reverses cisplatin resistance in gastric cancer through the PI3KAKT signaling pathway. Cancer Med 7:1369-1383. https://doi. org/10.1002/cam4.1380

38. Zheng WJ, Cao CW, Liu YN et al (2015) Multifunctional polyamidoamine-modified selenium nanoparticles dualdelivering siRNA and cisplatin to A549/DDP cells for reversal multidrug resistance. Acta Biomater 11:368-380. https:// doi.org/10.1016/j.actbio.2014.08.035

39. Zheng WJ, Yin TT, Chen QC et al (2016) Co-delivery of Se nanoparticles and pooled SiRNAs for overcoming drug resistance mediated by P-glycoprotein and class III $\beta$-tubulin in drugresistant breast cancers. Acta Biomater 31:197-210. https://doi. org/10.1016/j.actbio.2015.11.041

40. Ebrahimi S, Hosseini M, Shahidsales S et al (2017) Targeting the Akt/PI3K Signaling Pathway as a Potential Therapeutic Strategy for the Treatment of Pancreatic Cancer. Curr Med Chem 24:13211331. https://doi.org/10.2174/0929867324666170206142658

41. Winder A, Unno K, Yu Y et al (2017) The allosteric AKT inhibitor, MK2206, decreases tumor growth and invasion in patient derived xenografts of endometrial cancer. Cancer Biol Ther 18:958-964. https://doi.org/10.1080/15384047.2017.1281496

Publisher's Note Springer Nature remains neutral with regard to jurisdictional claims in published maps and institutional affiliations. 